\title{
THE STRUCTURE OF COMPOUNDED TRIVARIATE POISSON DISTRIBUTION
}

\author{
BY WEN-GI LIANG
}

\section{§. Summary.}

The compounded Poisson distribution in bivariate case is discussed in Kocherlakota [1], the method of introduction is compounding bivariate Poisson distribution $P(\tau \boldsymbol{\lambda})$ with a randomized parameter $\tau$. The author claimed that many distributions such as Poisson distribution itself, negative binomial and Hermite distribution etc are derived from the distribution.

The purpose of this paper is to generalize the method to trivariate case so as to get a uniform treatment of trivariate distributions, to get the structure of the distribution in detail and to get a clue of a generalization to multivariate distribution.

The compounded Poisson distribution is a family of distributions and it includes Poisson distribution itself. We could say the distribution is one kind of generalized Poisson. See, another generalization, Kawamura [2]. Also we will represent a differential formula of p.g.f. of the distribution and recurrence relations of p.d..

\section{$\S 2 . \quad$ Notations and Definitions.}

$\tau$ : compounding random variable, $-\infty<\tau<\infty$.

$g(\tau)$ : probability density function (p.d.f.) of $\tau$ or probability distribution (p. d.) of $\tau$.

$M(\theta)$ : moment generating function (m.g.f.) of $\tau$

$$
\begin{aligned}
M(\theta)=E\left(e^{\theta \tau}\right) & =\int g(\tau) e^{\theta \tau} d \tau \\
\text { or } \quad & =\sum_{\tau} g(\tau) e^{\theta \tau} .
\end{aligned}
$$

$\lambda=\left(\lambda_{1}, \lambda_{2}, \lambda_{3}, \lambda_{4}, \lambda_{5}, \lambda_{6}, \lambda_{7}\right):$ a parameter vector of trivariate Poisson distribution, $\lambda_{2} \geqq 0(i=1,2, \cdots, 7)$.

$P(\boldsymbol{\lambda})$ : trivariate Poisson distribution with parameter $\boldsymbol{\lambda}$.

$\Pi_{\tau}\left(Z_{1}, Z_{2}, Z_{3}\right)$ : probability generating function (p.g.f.) of trivariate Poisson distribution $P(\tau \lambda)$.

Received May 17, 1988 
$\Pi\left(Z_{1}, Z_{2}, Z_{3}\right):$ p.g.f. of compounded trivariate Poisson distribution.

$f_{r, s, t}=P(X=r, Y=s, Z=t)$ where $r, s, t \geqq 0$ integers

: probability distribution of compound trivariate Poisson distribution.

\section{$\S 3$. The structure of compounded trivariate Poisson distribution.}

Consider a trivariate Poisson distribution $P(\tau \lambda)$ for fixed nonnegative value $\tau$. Then $P(\tau \boldsymbol{\lambda})$ has a p.g.f.

$$
\begin{aligned}
\Pi_{\tau}\left(Z_{1}, Z_{2}, Z_{3}\right)=\exp \{\tau & {\left[\lambda_{1}\left(Z_{1}-1\right)+\lambda_{2}\left(Z_{2}-1\right)+\lambda_{3}\left(Z_{3}-1\right)+\lambda_{4}\left(Z_{1} Z_{2}-1\right)\right.} \\
& \left.\left.+\lambda_{5}\left(Z_{1} Z_{3}-1\right)+\lambda_{6}\left(Z_{2} Z_{3}-1\right)+\lambda_{7}\left(Z_{1} Z_{2} Z_{3}-1\right)\right]\right\} .
\end{aligned}
$$

Let us define $\tau$ a random variable with a p.d.f. $g(\tau)$ or p.d. $g\left(\tau_{\imath}\right)$ $(\imath=1,2, \cdots)$. And consider a mixed distribution of trivariate Poisson distribution $\int P(\tau \boldsymbol{\lambda}) g(\tau) d \tau$ or $\sum_{\imath} P\left(\tau_{i} \boldsymbol{\lambda}\right) g\left(\tau_{\imath}\right)$. We call this "a trivariate compounded Poisson distribution". P.g.f. of the distribution becomes

$$
\Pi\left(Z_{1}, Z_{2}, Z_{3}\right)=\int \Pi_{\tau}\left(Z_{1}, Z_{2}, Z_{3}\right) g(\tau) d \tau .
$$

That is, the p.g.f. of an expected p.g.f. concerning trivariate Poisson distribution $P(\tau \boldsymbol{\lambda})$. The distribution is the theme of this paper. In the later section it will be shown the compounded Poisson distribution consists many distributions such as Poisson distribution itself, negative binomial, Hermite and Neyman Type A etc.

Using the moment generating function (m.g.f.) of $\tau: M(\theta)=E\left(e^{\theta \tau}\right)=$ $\int g(\tau) e^{\theta \tau} d \tau$, we can derive the p.g.f. of the compounded Poisson distribution as following theorem.

THEOREM 1. The p.g.f. of compounded Poisson distribution is given by the m.g.f. $M(\theta)$ of $\tau$ replacing $\theta$ by $u=\lambda_{1}\left(Z_{1}-1\right)+\lambda_{2}\left(Z_{2}-1\right)+\lambda_{3}\left(Z_{3}-1\right)+\lambda_{4}\left(Z_{1} Z_{2}-1\right)$ $+\lambda_{5}\left(Z_{1} Z_{3}-1\right)+\lambda_{6}\left(Z_{2} Z_{3}-1\right)+\lambda_{7}\left(Z_{1} Z_{2} Z_{3}-1\right)$.

$$
\text { Proof. } \begin{aligned}
\Pi\left(Z_{1}, Z_{2}, Z_{3}\right) & =\int g(\tau) \Pi_{\tau}\left(Z_{1}, Z_{2}, Z_{3}\right) d \tau \\
& =\int g(\tau) \exp (\tau u) d \tau \\
& =M(u) .
\end{aligned}
$$

Furthermore we have a differential formula for the p.g.f. of the distribution. The formula helps us considerably many as deriving the probability density and analysis of the structure of the distribution. 
Let us use the notations $T_{\imath}, T_{\imath}$ for abbreviation as the differential operator of trivariate function $u$ with respect to $Z_{1}, Z_{2}, Z_{3}$ as following

$$
T_{\imath}=\frac{\partial u}{\partial Z_{\imath}} \quad(i=1,2,3) ; \quad T_{\imath \jmath}=\frac{\partial^{2} u}{\partial Z_{i} \partial Z_{\jmath}}=\frac{\partial^{2} u}{\partial Z_{j} \partial Z_{\imath}} \quad(i, j=1,2,3) .
$$

TheOREM 2. Let $\Pi\left(Z_{1}, Z_{2}, Z_{3}\right)$ be the p.g.f. of the compounded Poisson distribution. Then we have a differential formula of the distribution $\int P(\tau \lambda) g(\tau) d \tau$

$$
\begin{aligned}
& \frac{\partial^{r+s+t}}{\partial Z_{1}^{r} \partial Z_{2}^{s} \partial Z_{3}^{t}} \Pi\left(Z_{1}, Z_{2}, Z_{3}\right) \\
& =\sum_{[c]} \frac{r ! s ! t !}{a_{1} ! \cdots a_{7} !} M^{\left(\sum_{\imath=1}^{7} a_{\imath}\right)}{ }_{(u)} T_{1}^{a_{1}} T_{2}^{a_{2}} T_{3}^{a_{3}} T_{12}^{a_{4}} T_{13}^{a_{5}} T_{23}^{a_{2} \lambda_{7}^{\alpha} \lambda_{7}} .
\end{aligned}
$$

Where $r, s$ and $t$ are non-negative integers and $[c]$ means the set of vectors $\left(a_{1}, a_{2}, \cdots, a_{7}\right)$ satisfying $a_{1}+a_{4}+a_{5}+a_{7}=r, a_{2}+a_{4}+a_{6}+a_{7}=s, a_{3}+a_{5}+a_{6}+a_{7}=t$ and $a_{\imath}$ is non-negative integer for $i=1,2, \cdots, 7$.

Proof. Let us proceed to prove by the induction for $r, s$ and $t$. In the first step we should check the validity of the formula for $r=s=t=1$ and assuming that validity of it for some $r, s, t \geqq 1$ and if we could check the validity of it for $r+1, s, t$ or $r, s+1, t$ or $r, s, t+1$ then the induction will be completed as followings.

( I ) In the case of $r=s=t=1$. Left side of formula (3-2-1) becomes

$$
M^{\prime \prime \prime} T_{1} T_{2} T_{3}+M^{\prime \prime} T_{1} T_{23}+M^{\prime \prime} T_{2} T_{13}+M^{\prime \prime} T_{3} T_{12}+M^{\prime} \lambda_{7}
$$

and it consists with right side of the formula. This is the first step of the induction.

(II) Differentiate both sides of the formula by $Z_{1}$, we get

$$
\begin{aligned}
& \frac{\partial^{r+s+t+1}}{\partial Z_{1}^{r+1} \partial Z_{2}^{s} \partial Z_{3}^{t}} \Pi\left(Z_{1}, Z_{2}, Z_{3}\right) \\
& =\sum_{[c]} \frac{r ! s ! t !}{a_{1} ! \cdots a_{7} !} M^{\left(\left(\sum_{\imath=1}^{7} a_{\imath}\right)+1\right)}{ }_{(u)} T_{1}^{a_{1}+1} T_{2}^{a_{2}} T_{3}^{a_{3}} T_{12}^{a_{4}} T_{13}^{a_{5}} T_{23}^{a_{6}} \lambda_{7}^{a_{7}} \\
& +\sum \frac{r ! s ! t !}{a_{1} !\left(a_{2}-1\right) ! a_{3} ! \cdots a_{7} !} M^{\left(\sum_{\imath=1}^{7} a_{\imath}\right)}{ }_{(u)} T_{1}^{a_{1}} T_{2}^{a_{2}-1} T_{3}^{a_{3}} T_{12}^{a_{4}+1} T_{13}^{a_{5}} T_{23}^{\alpha_{6}} \lambda_{7}^{a_{7}} \\
& \left\{\begin{array}{l}
a_{1}+a_{4}+a_{5}+a_{7}=r \\
a_{2}+a_{4}+a_{6}+a_{7}=s \\
a_{3}+a_{5}+a_{6}+a_{7}+t \\
a_{2} \geqq 1, a_{2} \geqq 0 \quad(i=1,3,4, \cdots, 7) \text { non-negative integers }
\end{array}\right. \\
& +\sum \frac{r ! s ! t !}{a_{1} ! a_{2} !\left(a_{3}-1\right) ! a_{4} ! \cdots a_{7} !} M^{\left(\sum_{\imath=1}^{7} a_{\imath}\right)}{ }_{(u)} T_{1}^{a_{1}} T_{2}^{a_{2}} T_{3}^{a_{3}-1} T_{12}^{a_{4}} T_{13}^{a_{5}+1} T_{23}^{a_{6} \lambda_{7}^{a_{7}}}
\end{aligned}
$$




$$
\begin{aligned}
& \left\{\begin{array}{l}
a_{1}+a_{4}+a_{5}+a_{7}=r \\
a_{2}+a_{4}+a_{6}+a_{7}=s \\
a_{3}+a_{5}+a_{6}+a_{7}=t \\
a_{3} \geqq 1, \quad a_{\jmath} \geqq 0 \quad(j=1,2,4, \cdots, 7) \quad \text { non-negative integers }
\end{array}\right. \\
& +\Sigma \frac{r ! s ! t !}{a_{1} ! \cdots a_{5} !\left(a_{6}-1\right) ! a_{7} !} M^{\left(\sum_{2=1}^{7} a_{\imath}\right)}{ }_{(u)} T_{1}^{a_{1}} T_{2}^{a_{2}} T_{3}^{a_{3}} T_{12}^{a_{4}} T_{13}^{a_{5}} T_{23}^{a_{6}{ }^{-1}} \lambda^{\alpha_{7}+1} \\
& \left\{\begin{array}{l}
a_{1}+a_{4}+a_{5}+a_{7}=r \\
a_{2}+a_{4}+a_{6}+a_{7}=s \\
a_{3}+a_{5}+a_{6}+a_{7}=t \\
a_{6} \geqq 1, a_{k} \geqq 0 \quad(k=1,2, \cdots, 5,7) \text { non-negative integers. }
\end{array}\right.
\end{aligned}
$$

Let us denote four terms of the right side $(1) \sim(4)$, then we have

$$
\frac{\partial^{r+s+t+1}}{\partial Z_{1}^{r+1} \partial Z_{2}^{s} \partial Z_{3}^{t}} \Pi\left(Z_{1}, Z_{2}, Z_{3}\right)=(1)+(2)+(3)+(4) \text {. }
$$

Put $a_{1}+1=A_{1}$ then we have

$$
\begin{aligned}
(1)= & \sum \frac{r ! s ! t !}{\left(A_{1}-1\right) ! a_{2} ! \cdots a_{7} !} M^{\left(A_{1}+a_{2}+\cdot+a_{7}\right)}{ }_{(u)} T_{1}^{A_{1}} T_{2}^{a_{2}} T_{3}^{a_{3}} T_{12}^{a_{4}} T_{13}^{a_{5}} T_{23}^{a_{6}} \lambda_{7}^{a_{7}} \\
& \left\{\begin{array}{l}
A_{1}+a_{4}+a_{5}+a_{7}=r+1 \\
a_{2}+a_{4}+a_{6}+a_{7}=s \\
a_{3}+a_{5}+a_{6}+a_{7}=t \\
A_{1} \geqq 1, a_{\imath} \geqq 0 \quad(i=2,3, \cdots, 7) \quad \text { (non-negative integers. }
\end{array}\right.
\end{aligned}
$$

Put $A_{1}=a_{1}$ then we have

$$
\begin{aligned}
& \text { (1) }=\sum \frac{a_{1} r ! s ! t !}{a_{1} ! \cdots a_{7} !} M^{\left(\sum_{2=1}^{7} a_{2}\right)}{ }_{(u)} T_{1}^{a_{1}} T_{2}^{a_{2}} T_{3}^{a_{3}} T_{12}^{a_{4}} T_{13}^{a_{5}} T_{23}^{a_{6}} \lambda_{7}^{a_{7}} \\
& \left\{\begin{array}{l}
a_{1}+a_{4}+a_{5}+a_{7}=r+1 \\
a_{2}+a_{4}+a_{6}+a_{7}=s \\
a_{3}+a_{5}+a_{6}+a_{7}=t \\
a_{\imath} \geqq 0 \quad(i=1,2, \cdots, 7) \text { non-negative integers }
\end{array}\right. \\
& -\sum \frac{a_{1} r ! s ! t !}{a_{1} ! \cdots a_{7} !} M^{\left(\sum_{2=1}^{7} a_{2}\right)}{ }_{(u)} T_{1}^{a_{1}} T_{2}^{a_{2}} T_{3}^{a_{3}} T_{12}^{a_{4}} T_{13}^{a_{5}} T_{23}^{a_{6}} \lambda_{7}^{a_{7}} \\
& \left\{\begin{array}{l}
a_{1}+a_{4}+a_{5}+a_{7}=r+1 \\
a_{2}+a_{4}+a_{6}+a_{7}=s \\
a_{3}+a_{5}+a_{6}+a_{7}=t \\
a_{1}=0, a_{\imath} \geqq 0 \quad(i=2,3, \cdots, 7) \text { non-negative integers. }
\end{array}\right.
\end{aligned}
$$

Let us put two terms of the right side as above $(1)=\left(1^{\prime}\right)-\left(1^{\prime \prime}\right)$, we can easily check $\left(1^{\prime \prime}\right)=0$ and we have 
(1) $=\sum \frac{a_{1} r ! s ! t !}{a_{1} ! \cdots a_{7} !} M^{\left(\sum_{\imath=1}^{7} a_{2}\right)}{ }_{(u)} T_{1}^{a_{1}} T_{2}^{a_{2}} T_{3}^{a_{3}} T_{12}^{a_{4}} T_{13}^{a_{5}} T_{23}^{a_{6}} \lambda_{7}^{a_{7}}$
$\left[c^{\prime}\right]\left\{\begin{array}{l}a_{1}+a_{4}+a_{5}+a_{7}=r+1 \\ a_{2}+a_{4}+a_{6}+a_{7}=s \\ a_{3}+a_{5}+a_{6}+a_{7}=t \\ a_{\imath} \geqq 0 \quad(i=1,2, \cdots, 7) \text { non-negative integers. }\end{array}\right.$

Put $a_{2}-1=A_{2}, a_{4}+1=A_{4}$ then we have

$$
\begin{aligned}
(2)=\sum & \frac{r ! s ! t !}{a_{1} ! A_{2} ! a_{3} !\left(A_{4}-1\right) ! a_{5} ! a_{6} ! a_{7} !} M^{\left(a_{1}+A_{2}+a_{3}+A_{4}+a_{5}+a_{6}+a_{7}\right)} \\
& \left\{\begin{array}{l}
a_{1}+a_{4}+a_{5}+a_{7}=r+1 \\
a_{2}+a_{4}+a_{6}+a_{7}=s \\
a_{3}+a_{5}+a_{6}+a_{7}=t \\
a_{1}, A_{2}, a_{3}, a_{5}, a_{6}, a_{7} \geqq 0, A_{4} \geqq 1 \quad \times T^{a_{1}} T^{a_{2}} T^{a_{3}} T^{a_{4}} T^{a}
\end{array}\right.
\end{aligned}
$$

Put $A_{2}=a_{2}, A_{4}=a_{4}$ then we have

(2) $=\sum \frac{a_{4} r ! s ! t !}{a_{1} ! \cdots a_{7} !} M^{\left(\sum_{2=1}^{7} a_{2}\right)}{ }_{(u)} T_{1}^{a_{1}} T_{2}^{a_{2}} T_{3}^{a_{3}} T_{12}^{a_{4}} T_{13}^{a_{5}} T_{23}^{a_{6}} \lambda_{7}^{a_{7}}$

$$
\begin{aligned}
& {\left[c^{\prime}\right]\left\{\begin{array}{l}
a_{1}+a_{4}+a_{5}+a_{7}=r+1 \\
a_{2}+a_{4}+a_{6}+a_{7}=s \\
a_{3}+a_{5}+a_{6}+a_{7}=t \\
a_{2} \geqq 0 \quad(i=1,2, \cdots, 7) \text { non-negative integers }
\end{array}\right.} \\
& -\sum \frac{a_{4} r ! s ! t !}{a_{1} ! \cdots a_{7} !} M^{\left(\sum_{\imath=1}^{7} a_{\imath}\right)}{ }_{(u)} T_{1}^{a_{1}} T_{2}^{a_{2}} T_{3}^{a_{3}} T_{12}^{a_{4}} T_{13}^{a_{5}} T_{23}^{a_{6} 6 \lambda_{7}^{a_{7}}} \\
& \left\{\begin{array}{l}
a_{1}+a_{4}+a_{5}+a_{7}=r+1 \\
a_{2}+a_{4}+a_{6}+a_{7}=s \\
a_{3}+a_{5}+a_{6}+a_{7}=t \\
a_{4}=0, a_{2} \geqq 0 \quad(i=1,2,3,5,6,7) \text { non-negative integers. }
\end{array}\right.
\end{aligned}
$$

Let us put similarly as $(1),(2)=\left(2^{\prime}\right)-\left(2^{\prime \prime}\right)$, we can easily check $\left(2^{\prime \prime}\right)=0$ as $\left(1^{\prime \prime}\right)=0$ and we have

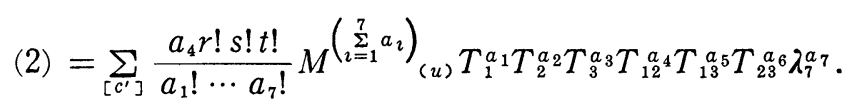

In the same way we have

$$
\text { (3) }=\sum_{\left[c^{\prime}\right]} \frac{a_{5} r ! s ! t !}{a_{1} ! \cdots a_{7} !} M^{\left(\sum_{2=1}^{7} a_{2}\right)}{ }_{(u)} T_{1}^{a_{1}} T_{2}^{a_{2}} T_{3}^{a_{3}} T_{12}^{a_{4}} T_{13}^{a_{5}} T_{23}^{a_{6}} \lambda_{7}^{a_{7}}
$$

and 
(4) $=\sum_{\left[c^{\prime}\right]} \frac{a_{7} r ! s ! t !}{a_{1} ! \cdots a_{7} !} M^{\left(\sum_{2=1}^{7} a_{\imath}\right)}{ }_{(u)} T_{1}^{a_{1}} T_{2}^{a_{2}} T_{3}^{a_{3}} T_{12}^{a_{4}} T_{13}^{a_{5}} T_{23}^{a_{3}} \lambda_{7}^{a_{7}}$.

Then we have

$$
\begin{aligned}
& \frac{\partial^{r+s+t+1}}{\partial Z_{1}^{r+1} \partial Z_{2}^{s} \partial Z_{3}^{t}} \Pi\left(Z_{1}, Z_{2}, Z_{3}\right)=(1)+(2)+(3)+(4) \\
& =\sum_{\left[c^{\prime}\right]} \frac{\left(a_{1}+a_{4}+a_{5}+a_{7}\right) r ! s ! t !}{a_{1} ! \cdots a_{7} !} M^{\left(\sum_{l=1}^{7} a_{\imath}\right)}{ }_{(u)} T_{1}^{a_{1}} T_{2}^{a_{2}} T_{3}^{a_{3}} T_{12}^{a_{4}} T_{13}^{a_{5}} T_{23}^{a_{6}} \lambda_{7}^{a_{7}} \\
& =\Sigma \frac{(r+1) ! s ! t !}{a_{1} ! \cdots a_{7} !} M^{\left(\sum_{\imath=1}^{7} a_{2}\right)}{ }_{(u)} T_{1}^{a_{1}} T_{2}^{a_{2}} T_{3}^{a_{3}} T_{12}^{a_{4}} T_{13}^{a_{5}} T_{23}^{a_{6}} \lambda_{7}^{a_{7}} \\
& {\left[c^{\prime}\right]\left\{\begin{array}{l}
a_{1}+a_{4}+a_{5}+a_{7}=r+1 \\
a_{2}+a_{4}+a_{6}+a_{7}=s \\
a_{3}+a_{5}+a_{6}+a_{7}=t \\
a_{\imath} \geqq 0 \quad(i=1,2, \cdots, 7) \text { non-negative integers. }
\end{array}\right.}
\end{aligned}
$$

In the same way by differentiation of the equation (3-2-3) with respect to $Z_{2}$ and $Z_{3}$, we can get following equation

$$
\begin{aligned}
& \frac{\partial^{r+s+t+3}}{\partial Z_{1}^{r+1} \partial Z_{2}^{s+1} \partial Z_{3}^{t+1}} \Pi\left(Z_{1}, Z_{2}, Z_{3}\right) \\
& =\Sigma \frac{(r+1) !(s+1) !(t+1) !}{a_{1} ! \cdots a_{7} !} M^{\left(\sum_{2=1}^{7} a_{2}\right)}{ }_{(u)} T_{1}^{a_{1}} T_{2}^{a_{2}} T_{3}^{a_{3}} T_{12}^{a_{4}} T_{13}^{a_{5}} T_{23}^{a_{6}} \lambda_{7}^{a_{7}} \\
& \\
& \left\{\begin{array}{l}
a_{1}+a_{4}+a_{5}+a_{7}=r+1 \\
a_{2}+a_{4}+a_{6}+a_{7}=s+1 \\
a_{3}+a_{5}+a_{6}+a_{7}=t+1 \\
a_{\imath} \geqq 0 \quad(i=1,2, \cdots, 7) \text { non-negative integers. }
\end{array}\right.
\end{aligned}
$$

We can conclude that the validity of differential formula by the induction for $r, s$ and $t$ just completed.

We can calculate the probability distribution of the compounded Poisson distribution by the formula in theorem 2 .

THEOREM 3. Let $f(r, s, t)$ be a probability distribution of the compounded Poisson distribution. Then we can represent

$$
\begin{aligned}
f(r, s, t) & =\left.\frac{1}{r ! s ! t !} \cdot \frac{\partial^{r+s+t}}{\partial Z_{1}^{r} \partial Z_{2}^{s} \partial Z_{3}^{t}} \Pi\left(Z_{1}, Z_{2}, Z_{3}\right)\right|_{Z_{1}=Z_{2}=Z_{3}=0} \\
& =\sum_{[c]} \frac{1}{a_{1} ! \cdots a_{7} !} M^{\left(\sum_{2=1}^{7} a_{2}\right)}{ }_{(u) \lambda_{1}^{a_{1}} \lambda_{2}^{a_{2}} \lambda_{3}^{a} \lambda_{4}^{a_{4}} \lambda_{5}^{a_{5}} \lambda_{6}^{a_{6}} \lambda_{7}^{a_{7}}}
\end{aligned}
$$

for every non-negative integers $r, s$ and $t$ where $u=-\sum_{i=1}^{7} \lambda_{i}$. 


\section{$\S 4$. Examples of the compounded Poisson distribution.}

$E X .1$. If compounding value satisfies $\tau=k$ (constant) then we have

p.d.f. of $\tau: g(\tau)=g(k)=1$,

m.g.f. of $\tau: M(\theta)=\sum_{\tau=k} g(\tau) e^{\theta \tau}=e^{k \theta}$,

p.g.f. of compounded trivariate Poisson distribution:

$$
\Pi\left(Z_{1}, Z_{2}, Z_{3}\right)=e^{k u}=M(u) .
$$

Put $b=\sum_{\imath=1}^{7} a_{\imath}$ then we have $M^{(b)}(u)=k^{b} e^{k u}$ and

$$
f(r, s, t)=\sum_{[c]} \frac{1}{a_{1} ! \cdots a_{7} !} e^{-k \lambda_{1}-k \lambda_{2} \cdots-k \lambda_{7}}\left(k \lambda_{1}\right)^{a_{1}} \cdots\left(k \lambda_{7}\right)^{a_{7}} .
$$

This is a trivariate Poisson distribution with parameter $k \lambda$ where $\lambda=\left(\lambda_{1}, \lambda_{2}, \cdots, \lambda_{7}\right)$.

$E X .2$. If compounding value $\tau$ has a $\chi^{2}$ distribution of freedom $2 \alpha$ then we have

p.d.f. of $\tau: g(\tau)= \begin{cases}\frac{1}{\Gamma(\alpha)} \tau^{\alpha-1}\left(\frac{\alpha}{m}\right)^{\alpha} \exp \left(-\frac{\alpha}{m} \tau\right) & \tau \geqq 0 \\ 0 & \tau<0,\end{cases}$

m.g.f. of $\tau: \quad M(\theta)=\int g(\tau) e^{\theta \tau} d \tau$

$$
\begin{aligned}
= & \int \frac{1}{\Gamma(\alpha)} \tau^{\alpha-1}\left(\frac{\alpha}{m}\right)^{\alpha} \exp \left[-\left(\frac{\alpha}{m}-\theta\right) \tau\right] d \tau \\
= & \left(\frac{\alpha}{m}\right)^{\alpha}\left(\frac{\alpha}{m}-\theta\right)^{-\alpha} \int \frac{1}{\Gamma(\alpha)} \tau^{\alpha-1}\left(\frac{\alpha}{m}-\theta\right)^{\alpha} \\
& \quad \times \exp \left[-\left(\frac{\alpha}{m}-\theta\right) \tau\right] d \tau \\
= & \left(1-\frac{m \theta}{\alpha}\right)^{-\alpha},
\end{aligned}
$$

p.g.f. of compounded Poisson distribution:

$$
\Pi\left(Z_{1}, Z_{2}, Z_{3}\right)=\left(1-\frac{m u}{\alpha}\right)^{-\alpha}=M(u) .
$$

Put $\sum_{\imath=1}^{7} a_{\imath}=b,-\sum_{\imath=1}^{7} \lambda_{\imath}=\nu$ then we have

$$
M^{(b)}(u)=\left(-\frac{m}{\alpha}\right)^{b}(-\alpha)(-\alpha-1) \cdots(-\alpha-b+1)\left(1-\frac{m}{\alpha} u\right)^{-\alpha-b}
$$




$$
\begin{aligned}
& =\left(\frac{m}{\alpha}\right)^{b} \alpha(\alpha+1) \cdots(\alpha+b-1)\left(1-\frac{m}{\alpha} u\right)^{-\alpha-b} \\
& =\left(\frac{m}{\alpha}\right)^{b} \frac{\Gamma(\alpha+b)}{\Gamma(\alpha)}\left(1-\frac{m}{\alpha} u\right)^{-(\alpha+b)}
\end{aligned}
$$

and

$$
f(r, s, t)=\sum_{[c]} \frac{1}{a_{1} ! \cdots a_{7} !}\left(\frac{m}{\alpha}\right)^{b} \frac{\Gamma(\alpha+b)}{\Gamma(\alpha)}\left(1-\frac{m}{\alpha} \nu\right)^{-(\alpha+b)} \lambda_{1}^{a_{1}} \lambda_{2}^{a_{2}} \cdots \lambda_{7}^{a_{7}} .
$$

It is called trivariate Negative Binomial distribution.

$E X .3$. If compounding value $\tau$ has a Gaussian distribution, then we have

p.d.f. of $\tau: g(\tau)=\frac{1}{\sqrt{2 \pi} \sigma} \exp \left(-\frac{(\tau-\mu)^{2}}{2 \sigma^{2}}\right)$

m.g.f. of $\tau: \quad M(\theta)=\int \frac{1}{\sqrt{2 \pi} \sigma} \exp \left[-\frac{(\tau-\mu)^{2}}{2 \sigma^{2}}\right] e^{\theta \tau} d \tau$

$$
\begin{aligned}
& =\int \frac{1}{\sqrt{ } 2 \pi \sigma} \exp \left[-\frac{\tau^{2}-2\left(\mu+\theta \sigma^{2}\right) \tau+\mu^{2}}{2 \sigma^{2}}\right] d \tau \\
& =\exp \left(\mu \theta+\frac{\sigma^{2} \theta^{2}}{2}\right),
\end{aligned}
$$

p.g.f. of compounded Poisson distribution becomes :

$$
\Pi\left(Z_{1}, Z_{2}, Z_{3}\right)=\exp \left(\mu u+\frac{\sigma^{2}}{2} u^{2}\right)=M(u)
$$

Put $\sum_{i=1}^{7} a_{\imath}=b,-\sum_{i=1}^{7} \lambda_{\imath}=\nu$ then we have $M^{(b)}(u)=M(u) P_{b}(u)$ where $P_{b}(u)$ is a polynomial of degree $b$. Then we have

$$
f(r, s, t)=\sum_{[c]} \frac{1}{a_{1} ! \cdots a_{7} !} M(\nu) P_{b}(\nu) \lambda_{1}^{a_{1}} \lambda_{2}^{a_{2}} \cdots \lambda_{7}^{a_{7}} .
$$

It is called a trivariate Hermite distribution, see KocherLakota [1].

$E X .4$. If compounding value $\tau$ has a Poisson distribution, then we have

p.d.f. of $\tau: g(\tau)=\frac{\lambda^{\tau}}{\tau !} e^{-\lambda} \quad \tau \geqq 0$,

m.g.f. of $\tau: \quad M(\theta)=\int \frac{\lambda^{\tau}}{\tau !} e^{-\lambda} \cdot e^{\theta \tau} d \tau$

$$
\begin{aligned}
& =e^{\lambda\left(e^{\theta}-1\right)} \int \frac{\left(\lambda e^{\theta}\right)^{\tau} e^{-\lambda e^{\theta}}}{\tau !} d \tau \\
& =e^{\lambda\left(e^{\theta}-1\right)},
\end{aligned}
$$


p.g.f. of compounded Poisson distribution:

$$
\Pi\left(Z_{1}, Z_{2}, Z_{3}\right)=\exp \left[\lambda\left(e^{u}-1\right)\right]=M(u) .
$$

Put $\sum_{i=1}^{7} a_{\imath}=b,-\sum_{i=1}^{7} \lambda_{\imath}=\nu$ then we have $M^{(b)}(u)=\exp \left[\lambda\left(e^{u}-1\right)\right] \cdot m_{b}(u)$ where $m_{b}(u)$ is the degree $b$ moment of Poisson distribution $P\left(\lambda e^{u}\right)$ then we have

$$
f(r, s, t)=\sum_{[c]} \frac{1}{a_{1} ! \cdots a_{7} !} \exp \left[\lambda\left(e^{\nu}-1\right)\right] m_{b}(\nu) \lambda_{1}^{a_{1}} \lambda_{2}^{a_{2}} \cdots \lambda_{7}^{a_{7}}
$$

where $m_{b}(\nu)$ is the power $b$ moment of Poisson distribution $P\left(\lambda e^{\nu}\right)$. It is called a trivariate Neyman Type A distribution.

$E X .5$. If compounding value $\tau$ has a Inverse-Gaussian distribution, then we have

$$
\begin{aligned}
& \text { p.d.f. of } \tau: g(\tau)=\left(\frac{\lambda}{2 \pi \tau^{3}}\right)^{1 / 2} \exp \left[-\frac{\lambda}{2 \mu^{2} \tau}(\tau-\mu)^{2}\right] \quad \tau>0 \text {, } \\
& \text { m.g.f. of } \tau \text { : } \\
& M(\theta)=\int g(\tau) e^{\theta \tau} d \tau \\
& =\int\left(\frac{\lambda}{2 \pi \tau^{3}}\right)^{1 / 2} \exp \left[-\frac{\lambda}{2 \mu^{2} \tau}(\tau-\mu)^{2}+\theta \tau\right] d \tau . \\
& =\int\left(\frac{\lambda}{2 \pi \tau^{3}}\right)^{1 / 2} \exp \left[-\frac{\lambda}{2 \mu^{2} \tau}\left(\tau^{2}-2 \tau \mu+\mu^{2}-\frac{2 \mu^{2} \theta}{\lambda} \tau^{2}\right)\right] d \tau \\
& =\int\left\{\frac{\lambda\left(1-\frac{2 \mu^{2}}{\lambda} \theta\right)^{1 / 2}}{2 \pi\left[\left(1-\frac{2 \mu^{2}}{\lambda} \theta\right)^{1 / 2} \tau\right]^{3}}\right\}^{1 / 2} \exp \left\{-\frac{\lambda\left(1-\frac{2 \mu^{2}}{\lambda} \theta\right)^{1 / 2}}{2 \mu^{2}\left(1-\frac{2 \mu^{2}}{\lambda} \theta\right)^{1 / 2} \tau}\right. \\
& \times\left\{\left[\left(1-\frac{2 \mu^{2}}{\lambda} \theta\right)^{1 / 2} \tau\right]^{2}-2\left(1-\frac{2 \mu^{2}}{\lambda} \theta\right)^{1 / 2} \tau \mu+\mu^{2}\right. \\
& \left.\left.-2\left[1-\left(1-\frac{2 \mu^{2} \theta}{\lambda}\right)^{1 / 2}\right] \tau \mu\right\}\right\} d\left(1-\frac{2 \mu^{2}}{\lambda} \theta\right)^{1 / 2} \tau \\
& =\exp \frac{\lambda}{\mu}\left[1-\left(1-\frac{2 \mu^{2}}{\lambda} \theta\right)^{1 / 2}\right] \text {. }
\end{aligned}
$$

p.g.f. of compounded Poisson distribution:

$$
\Pi\left(Z_{1}, Z_{2}, Z_{3}\right)=\exp \frac{\lambda}{\mu}\left[1-\left(1-\frac{2 \mu^{2}}{\lambda} u\right)^{1 / 2}\right]=M(u) \text {. }
$$

Put $\sum_{\imath=1}^{7} a_{\imath}=b,-\sum_{\imath=1}^{7} \lambda_{\imath}=\nu$ then we have 


$$
M^{(b)}(u)=M(u) \mu^{b} \sum_{k=0}^{b-1} C_{k}\left(\frac{\mu}{\lambda}\right)^{k}\left(1-\frac{2 \mu^{2}}{\lambda} u\right)^{-b / 2-k / 2},
$$

where $C_{k}$ is a constant value. Then we have

$$
f(r, s, t)=\sum_{[c]} \frac{1}{a_{1} ! \cdots a_{7} !} M(\nu) \mu^{b} \sum_{k=0}^{b-1} C_{k}\left(\frac{\mu}{\lambda}\right)^{k}\left(1-\frac{2 \mu^{2}}{\lambda} \nu\right)^{-b / 2-k / 2} \lambda_{1}^{a_{1}} \lambda_{2}^{a_{2}} \cdots \lambda_{7}^{a_{7}} .
$$

It is called a trivariate Poisson Inverse-Gaussian distribution.

\section{$\S 5$. Discussion.}

We can derive recurrence relations of the probability distribution as following theorem. It will help us to calculate the probability distribution.

THEOREM 4. Let's assume $r \geqq 1, s \geqq 1, t \geqq 1$, then we have

1) $s \lambda_{1} f_{r, s, t}=(r+1) \lambda_{2} f_{r+1, s-1, t}+(r-s+1) \lambda_{4} f_{r, s-1, t}+(r+1) \lambda_{6} f_{r+1, s-1, t-1}$

$$
-s \lambda_{5} f_{r, s, t-1}+(r-s+1) \lambda_{7} f_{r, s-1, t-1} \text {. }
$$

2) $r \lambda_{2} f_{r, s, t}=(s+1) \lambda_{1} f_{r-1, s+1, t}+(s-r+1) \lambda_{4} f_{r-1, s, t}+(s+1) \lambda_{5} f_{r-1, s+1, t-1}$

$$
-r \lambda_{6} f_{r, s, t-1}+(s-r+1) \lambda_{7} f_{r-1, s, t-1} \text {. }
$$

3) $t \lambda_{1} f_{r, s, t}=(r+1) \lambda_{3} f_{r+1, s, t-1}+(r-t+1) \lambda_{5} f_{r, s, t-1}+(r+1) \lambda_{6} f_{r+1, s-1, t-1}$

$$
-t \lambda_{4} f_{r, s-1, t}+(r-t+1) \lambda_{7} f_{r, s-1, t-1} \text {. }
$$

4) $r \lambda_{3} f_{r, s, t}=(t+1) \lambda_{1} f_{r-1, s, t+1}+(t-r+1) \lambda_{5} f_{r-1, s, t}+(t+1) \lambda_{4} f_{r-1, s-1, t+1}$

$$
-r \lambda_{6} f_{r, s-1, t}+(t-r+1) \lambda_{7} f_{r-1, s-1, t} \text {. }
$$

5) $t \lambda_{2} f_{r, s, t}=(s+1) \lambda_{3} f_{r, s+1, t-1}+(s-t+1) \lambda_{6} f_{r, s, t-1}+(s+1) \lambda_{5} f_{r-1, s+1, t-1}$

$$
-t \lambda_{4} f_{r-1, s, t}+(s-t+1) \lambda_{7} f_{r-1, s, t-1} \text {. }
$$

6) $s \lambda_{3} f_{r, s, t}=(t+1) \lambda_{2} f_{r, s-1, t+1}+(t-s+1) \lambda_{6} f_{r, s-1, t}+(t+1) \lambda_{4} f_{r-1, s-1, t+1}$

$$
-s \lambda_{5} f_{r-1, s, t}+(t-s+1) \lambda_{7} f_{r-1, s-1, t} \text {. }
$$

The author will represent a paper concerning the relations of the compounded Poisson distribution in near future. The proof of this theorem will be included in the next paper.

\section{§ 6. Acnowledgement.}

The author expresses his hearty thanks to Professor K. Kawamura for his kind and continuous guidance during the progress of this work. Not only that, he helps the author understand Japanese texts, papers and spends much of his valuable time to look after personal problems as well. The author knows no word to express his gratitude to him. 


\section{REFERENCES}

[1] S. Kocherlakota, On the compounded bivariate Poisson distribution: A unified treatment. Annals of Institute of Mathematical Statistics, vol. 40 (1988), no. 1, 61-76.

[2] K. Kawamura, A generalization of multivariate Poisson distribution, Kodai Math. Jour. 3 (1980), 429-441.

Department of Mathematics

TOKyo Institute of TECHNOLOGy 